\title{
Integral and fractional charge filling in a InAs/GaAs quantum dot $p-i-n$ diode by capacitance-voltage measurement
}

\author{
Shiang-Feng Tang, Shih-Yen Lin, and Si-Chen Lee ${ }^{\mathrm{a})}$ \\ Department of Electrical Engineering and Graduate Institute of Electronic Engineering, National Taiwan \\ University, Taipei, Taiwan, Republic of China \\ Ya-Tung Cherng \\ Materials and Electro-Optics Division, Chung Shang Institute of Science and Technology, Lung Tan, \\ Taoyuan 325, Taiwan, Republic of China
}

(Received 13 August 2001; accepted for publication 13 February 2002)

\begin{abstract}
The temperature-dependent capacitance-voltage $(C-V)$ characteristics of two stacked InAs/GaAs quantum dot $p-i-n$ diode were investigated. The capacitance discontinuities observed are attributed to charge storage in the InAs quantum dots. The average storage electrons at each InAs quantum dot thus obtained are two and three electrons at room temperature and at temperature below $100 \mathrm{~K}$, respectively. In the intermediate temperature range from 100 to $250 \mathrm{~K}$, fractional charge occupation is observed in each dot. When the $C-V$ measurement frequency is lowered from 800 to $80 \mathrm{kHz}$, the capacitance turns into a negative value under low biases which indicates the dominance of the inductance at lower frequency. (C) 2002 American Institute of Physics.
\end{abstract}

[DOI: $10.1063 / 1.1467961]$

\section{INTRODUCTION}

The quantum dot (QD) nanostructure has attracted much attention recently due to its interesting optical and electrical properties. Many applications such as a high temperature operated quantum dot infrared photodetector (QDIP), ${ }^{1-7}$ lasers, ${ }^{8-10}$ memory devices ${ }^{11-13}$ and other electronic devices ${ }^{14}$ have been investigated. It was found that the capacitance-voltage $(C-V)$ measurement was an useful technique by which to study the charge distribution in QDs. ${ }^{15}$ For example, the complicated spatial charge distribution of InAs/GaAs quantum dots embedded in a $p-n$ junction due to the Coulomb interaction between electrons in the QDs and bulk GaAs was derived from its $C-V$ characteristics. ${ }^{15,16}$ These data could be applied to numerical simulations and determined the energy levels of QDs in a Schottky diode. ${ }^{17}$

In this article, to further investigate this phenomenon, a two-stacked InAs/GaAs QD pin structure is prepared by molecular beam epitaxy (MBE). A model was developed based on the self-consistent solution of the Poisson equation for bipolar transport ${ }^{18}$ and an $R L C$ equivalent circuit was established to extract the intrinsic capacitance in a $p n$ diode. Also observed were the temperature-dependent $C-V$ characteristics of the $p-i-n$ diode ranging from 20 to $300 \mathrm{~K}$. The hysteresis and capacitance discontinuity observed were attributed to the charge filling effect of the InAs QDs embedded in the $p-i-n$ diode. The average storage electrons in each InAs QD were two and three electrons at room temperature and at temperature below $100 \mathrm{~K}$, respectively. In the intermediate temperature range from 100 to $250 \mathrm{~K}$, fractional charge occupation was observed in each dot.

a)Electronic mail: sclee@cc.ee.ntu.edu.tw

\section{EXPERIMENTS}

A two stacked InAs/GaAs QD $p-i-n$ structure was grown by a VG $80 \mathrm{H}$ solid-source MBE system. The device structure is shown in Fig. 1. Sandwiched between $0.5 \mu \mathrm{m}$ $p$-GaAs and $1 \mu \mathrm{m} n$-GaAs two undoped InAs QD layers with 3 monolayer (ML) InAs coverage and three $50 \mathrm{~nm}$ undoped GaAs barriers are grown on a (100) $N$-type GaAs substrate at $510{ }^{\circ} \mathrm{C} .{ }^{9}$ The doping concentrations of the $p$-type and $n$-type GaAs contact layers are $5 \times 10^{18}$ and 1 $\times 10^{18} \mathrm{~cm}^{-3}$, respectively. The InAs quantum dots are grown at $0.5 \mathrm{ML} / \mathrm{s}$ under an arsenic shutter closed condition. The dot density measured by the atomic-force microscopy is about $4 \times 10^{10} \mathrm{~cm}^{-2}$.

A two-terminal device is fabricated using standard processes. The device area is patterned to about 3.68 $\times 10^{-4} \mathrm{~cm}^{2}$. With $\mathrm{Au} / \mathrm{Ge} / \mathrm{Ni}$ and Au sequentially evaporated on the bottom $n$-type GaAs substrate and $\mathrm{Au} / \mathrm{Be}$ and $\mathrm{Au}$ on the top $p$-type contact layer, the sample is annealed at $410{ }^{\circ} \mathrm{C}$ for $60 \mathrm{~s}$ to form ohmic contact. The device is loaded into a CTI cryogenic system, the temperature-dependent dark

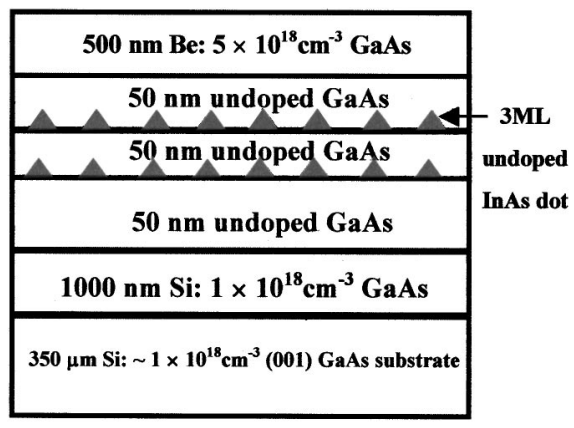

FIG. 1. Schematic diagram of the $p-i-n$ diode showing the insertion of two stacked InAs/GaAs dot layers in the $i$ region. 


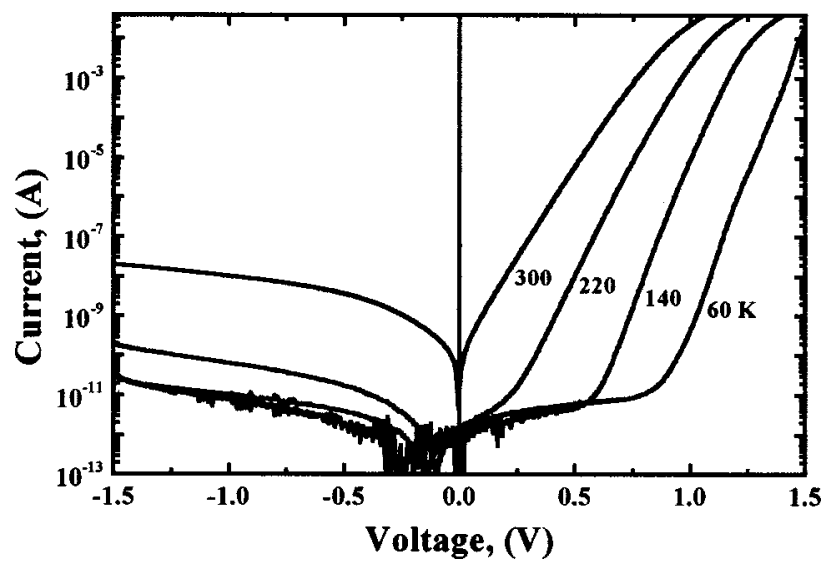

FIG. 2. Dark $I-V$ characteristics of the InAs/GaAs QD $p-i-n$ diode at different temperatures.

current-voltage $I-V$ and $C-V$ characteristics are measured by an HP4156B precision semiconductor parametric analyzer and an HP4284A precision low capacitance resonator (LCR) meter, respectively. The measurement equipment is carefully calibrated to compensate for parasitic capacitance and inductance.

\section{RESULTS AND DISCUSSION}

Figure 2 displays the dark $I-V$ characteristics of the two stacked InAs/GaAs QD $p-i-n$ diode measured at temperatures ranging from 60 to $300 \mathrm{~K}$. Both forward and reverse characteristics are shown. Figure 3 shows the dark current $I_{d}$ versus inverse temperature $1 / T$ curves under constant reverse biases. Two distinct regions are observed in Fig. 3 and the transition region lies between 100 and $180 \mathrm{~K}$. This phenomenon indicates that two different transport mechanisms are responsible for $I-V$ characteristics under reverse bias: first, the thermally activated minority carrier injection or generation process at temperature above $180 \mathrm{~K}$ and, second, the temperature-insensitive tunneling current at temperature below $100 \mathrm{~K}$. The band structures of the two stacked InAs/ GaAs QD $p-i-n$ diode under different biases are shown in Figs. 4(a)-4(c), respectively. The forward voltage applied reduces the potential barrier between the $p$ - and $n$-type re-

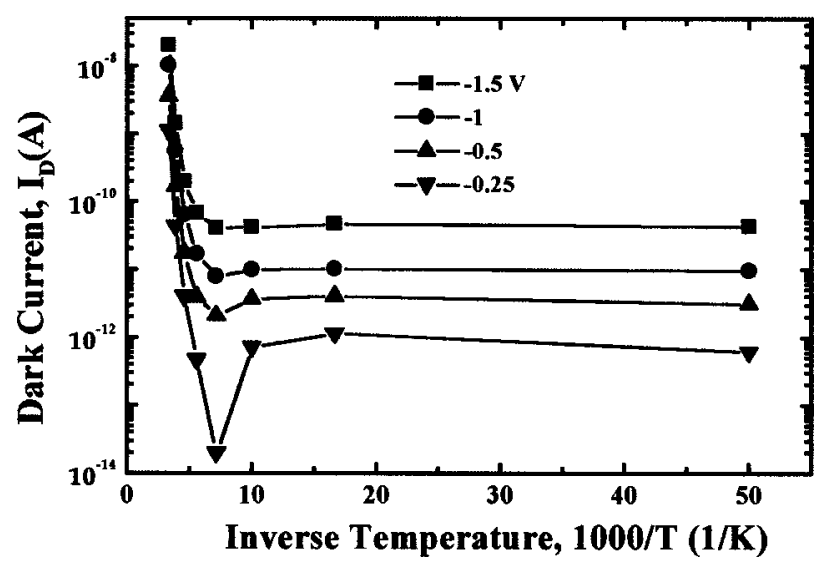

FIG. 3. Dark current $I_{d}$ as a function of inverse temperature at different bias voltages.

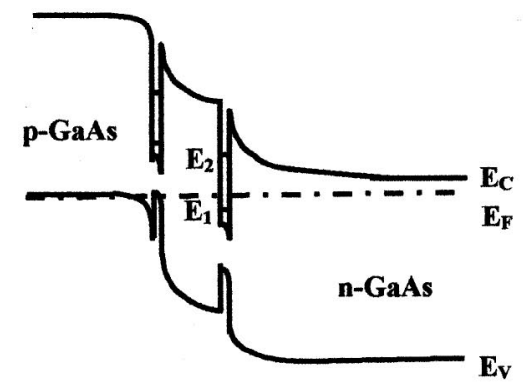

Undoped InAs/GaAs QD Structure

(a)

Zero bias

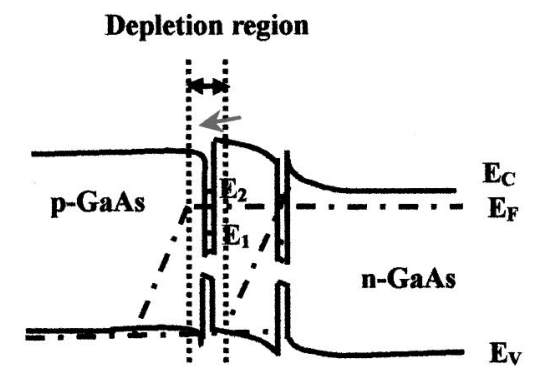

(b)

Forward bias

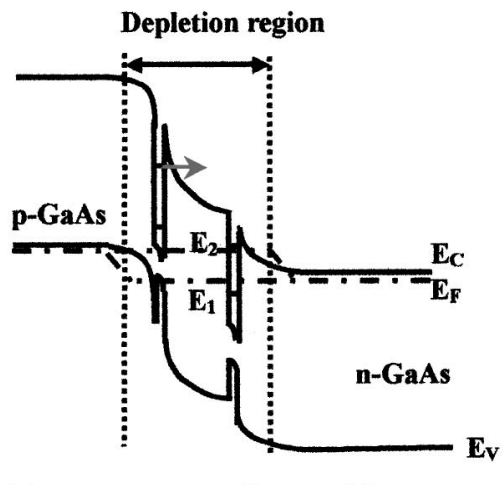

(c)

Reverse bias

FIG. 4. Energy band diagram under (a) zero, (b) forward and (c) reverse bias voltages, respectively. The gray arrows indicate the charging and discharging processes.

gions from the built-in voltage, $V_{\mathrm{bi}}$, to $V_{\mathrm{bi}}-V$, where $V$ is the voltage applied. Hence, the difference of the quasi-Fermi level in the depletion region is equal to $q V$ as shown in Fig. 4(b). ${ }^{19-21}$ With an increase in reverse bias, almost all the voltage applied has fallen across the depletion region such that the high electric field causes an asymmetrical energy band diagram in the InAs QD structure as shown in Fig. 4(c).

The $C-V$ characteristics of the two stacked InAs/GaAs QD $p-i-n$ diode are measured under modulation frequencies of 80 and $800 \mathrm{kHz}$ as shown in Figs. 5 and 6, respectively. As shown in Fig. 5, the capacitance decreases and turns into a negative value when the biases change from reverse to forward voltage. This indicates the dominance of the inductance at $80 \mathrm{kHz}$. However, no negative capacitance is observed for the measuring frequency at $800 \mathrm{kHz}$ as shown in Fig. 6. The capacitances measured at two frequencies exhibit staircase discontinuity and form hysteresis loops. With the voltages applied swept from -1 to $1 \mathrm{~V}$ and then 


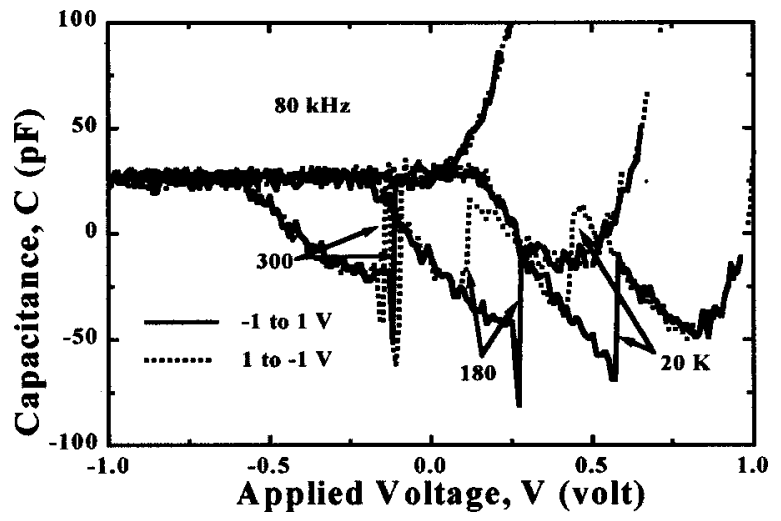

FIG. 5. Temperature-dependent $C-V$ characteristics measured at $80 \mathrm{kHz}$. The solid and dotted lines indicate the voltage-scanning direction from -1 to $1 \mathrm{~V}$ and from 1 to $-1 \mathrm{~V}$, respectively. The device area is 3.7 $\times 10^{-4} \mathrm{~cm}^{2}$.

back from 1 to $-1 \mathrm{~V}$, the hysteresis loops appear in the capacitance discontinuity regions under the low bias level, i.e., $<0.55 \mathrm{~V}$, and are attributed to the charging and discharging effect of electrons in the InAs QD structure. Also observed in the $C-V$ curves is that hysteresis loops and capacitance discontinuities are shifted toward lower or negative bias regions with an increase in temperature. This is because, at higher temperature, the background carrier concentration is higher in the $i$ layer, the depletion region starts to retreat from the $n^{+}$GaAs layer under lower bias. In the larger reverse bias regions, depletion-layer capacitance determined by the $i$-layer thickness accounts for most of the junction capacitance in the QD $p-i-n$ diode.

In order to model the $C-V$ characteristics under different modulation frequencies, the quantum dot structure is modeled with an $R-L-C$ network with interconnecting capacitance around the QDs, shown in Fig. 7. Its effective terminal admittance can be expressed as

$$
Y=\frac{R_{s}}{R_{s}^{2}+\left(\omega L_{s}\right)^{2}}+j \omega\left[C_{i}-\frac{L_{s} / R_{s}^{2}}{1+\left(\omega L_{s} / R_{s}\right)^{2}}\right]=G+j D,
$$

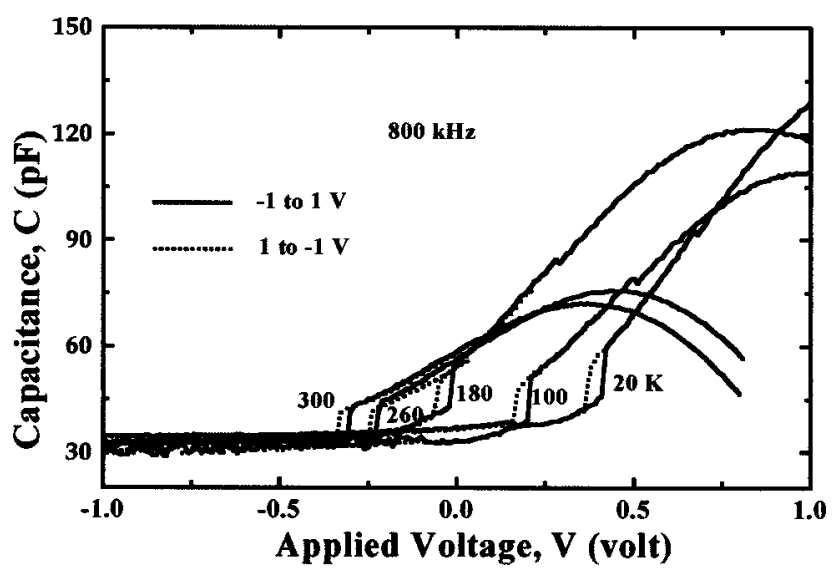

FIG. 6. Temperature-dependent $C-V$ characteristics measured at $800 \mathrm{kHz}$. The solid and dotted lines indicate the voltage-scanning direction from -1 to $1 \mathrm{~V}$ and from 1 to $-1 \mathrm{~V}$, respectively. The device area is 3.7 $\times 10^{-4} \mathrm{~cm}^{2}$.

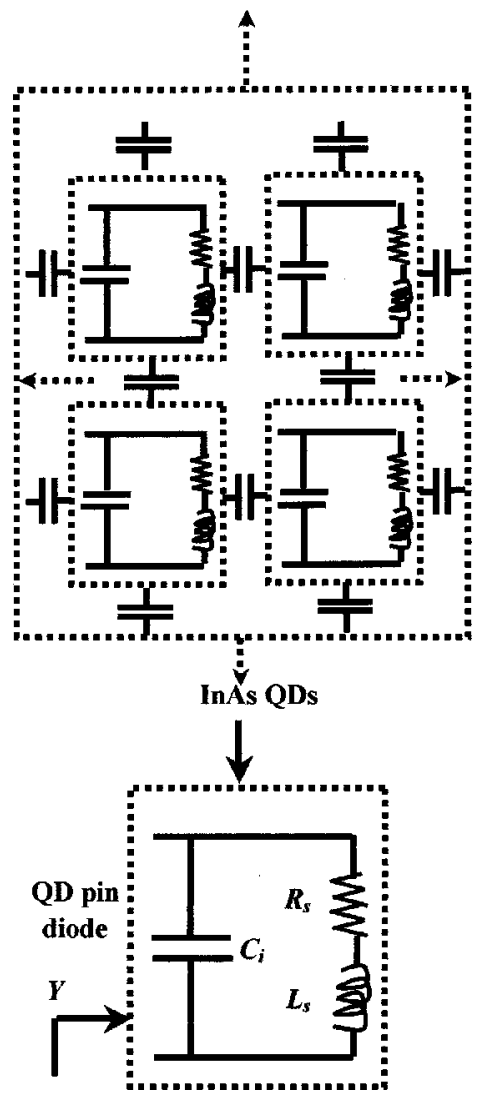

FIG. 7. $R-L-C$ equivalent circuit of the QD $p-i-n$ diode.

where $C_{i}, R_{s}$ and $L_{s}$ are the intrinsic capacitance, shunt resistance and inductance, respectively. For small-signal approximation, ac current $I=Y V=(G+j D) V, D=\omega C_{\text {eterm }}$, it turn out $C_{\text {eterm }}=\left\{C_{i}-L_{s} / R_{s}^{2} /\left[1+\left(\omega L_{s} / R_{s}\right)^{2}\right]\right\}$, where $C_{\text {eterm }}$ is the effective terminal capacitance. ${ }^{22}$ By obtaining suitable values of $C_{i}, R_{s}$ and $L_{s}$, with lower resonance frequency, $\omega$, a negative $C_{\text {eterm }}$ value can be obtained. Thus, the negative capacitance ${ }^{23}$ at lower measuring frequency $(<\sim 80 \mathrm{kHz})$ shown in Fig. 5 can be explained. The inductance may come from the QD property that the bias voltage and thus the electric field change induce a change in conduction current by charging and discharging the QDs. Therefore, voltage is phase leading the current which is the characteristic of inductance. Based on the above equation, $C_{\text {eterm }} \approx C_{i}$ under higher frequency. $\Delta C$ (capacitance discontinuity) can be extracted from temperature dependent $C-V$ characteristics measured by $800 \mathrm{kHz}$ and the result is shown in Fig. 8(a). The measurements start from $-1 \mathrm{~V}$ sweeping to $1 \mathrm{~V}$, and the depletion region is shortened abruptly at a certain bias, indicating electron filling in the QDs near the $n$-type contact. When the bias is sweeping from 1 back to $-1 \mathrm{~V}$, the discharging process is dominated by the electron tunneling effect in QDs under large reverse bias, ${ }^{24}$ as shown by the gray arrows in Fig. 4(c). The obvious staircase characteristics reveal the good electron charging uniformity in QDs. The average charge number in each dot is thus obtained and is shown in Fig. 8(b). It is clear that, below $100 \mathrm{~K}$, each QD can be charged with three electrons when the depletion region retreats from the QD region. And beyond $250 \mathrm{~K}$, each 


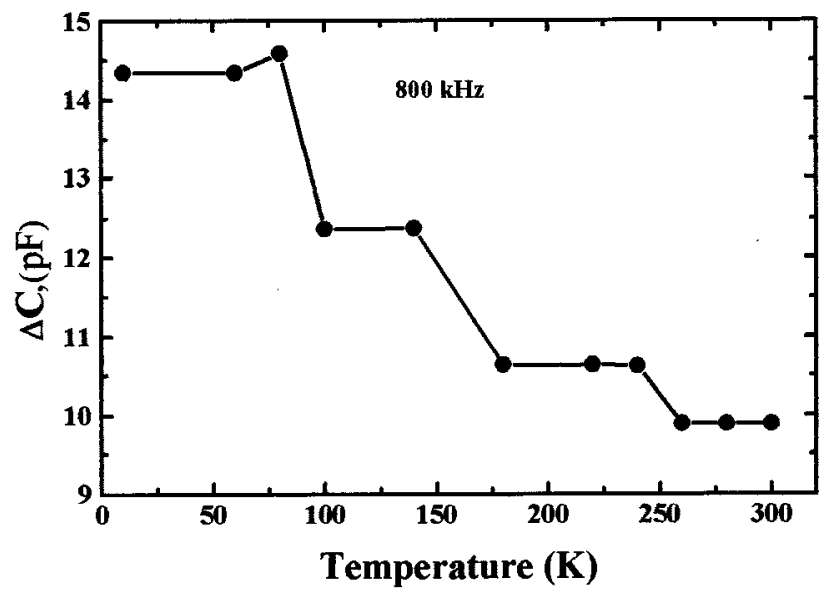

(a)

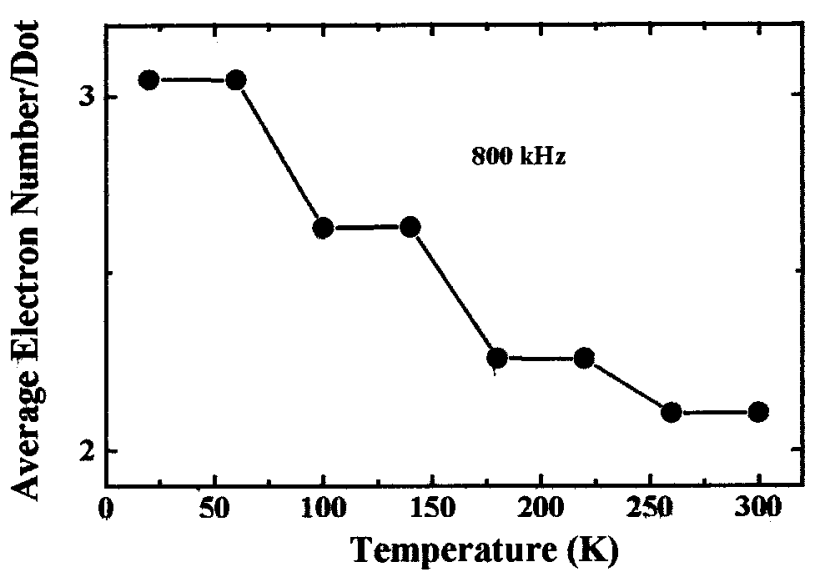

(b)

FIG. 8. (a) Capacitance discontinuity measured at different temperatures. (b) Number of storage electrons in each InAs QD at different temperatures.

QD is charged with two electrons. Certain fractional charges between two and three electrons are observed between 100 and $250 \mathrm{~K}$. This may be caused by two groups of QDs with different size distributions.

\section{CONCLUSIONS}

We have established a simplified $R-L-C$ circuit model to simulate the behavior of a two stacked InAs/GaAs QD $p-i-n$ diode. Negative capacitance was observed at a measuring frequency of $80 \mathrm{kHz}$, and is attributed to phase lagging of the conduction current to the bias voltage. The capacitance discontinuity can be used to extract the electron storage number in each dot. The average storage electrons at each InAs quantum dot thus obtained are two and three electrons at temperature higher than $250 \mathrm{~K}$ and at temperature below $100 \mathrm{~K}$, respectively. Fractional charge occupation is observed in the intermediate temperature range of $100-250 \mathrm{~K}$.

\section{ACKNOWLEDGMENT}

This work was supported by the National Science Council of the Republic of China under Contract No. NSC 902215-E-002-018.

${ }^{1}$ T. Cho, J.-W. Kim, J.-E. Oh, and S. Hong, Tech. Dig. - Int. Electron Devices Meet. 1998, 441.

${ }^{2}$ S. Y. Wang, S. D. Lin, H. W. Wu, and C. P. Lee, Appl. Phys. Lett. 78, 1023 (2001)

${ }^{3}$ J. Phillips, P. Bhattacharya, S. W. Kennerly, D. W. Beekman, and M. Dutta, IEEE J. Quantum Electron. 35, 936 (1999).

${ }^{4}$ D. Pan, E. Towe, and S. Kennerly, Appl. Phys. Lett. 75, 2719 (1999).

${ }^{5}$ S.-F. Tang, S.-Y. Lin, S.-C. Lee, C. H. Kuan, and Y.-T. Cherng, Tech. Dig. - Int. Electron Devices Meet. 2000, 601.

${ }^{6}$ S.-F. Tang, S.-Y. Lin, and S.-C. Lee, Appl. Phys. Lett. 78, 2428 (2001).

${ }^{7}$ S.-Y. Lin, Y.-R. Tsai, and S.-C. Lee, Appl. Phys. Lett. 78, 2784 (2001).

${ }^{8}$ D. Bimberg, M. Grundmann, and N. N. Ledentsov, Quantum Dot Heterostructures (Wiley, Germany, 1999), p. 297.

${ }^{9}$ N. Kirstaedter et al., Appl. Phys. Lett. 69, 1226 (1996).

${ }^{10}$ M. V. Maximov et al., J. Appl. Phys. 83, 5561 (1998).

${ }^{11}$ J. J. Finley, M. Skalitz, M. Arzberger, A. Zrenner, G. Böhm, and G. Abstreiter, Appl. Phys. Lett. 73, 2618 (1998).

${ }^{12}$ M. C. Bödefeld, R. J. Warburton, K. Karrai, J. P. Kotthaus, G. MedeirosRibeiro, and P. M. Petroff, Appl. Phys. Lett. 74, 1839 (1999).

${ }^{13}$ T. Lundstrim, W. Schoenfeld, H. Lee, and P. M. Petroff, Appl. Phys. Lett. 74, 1839 (1999).

${ }^{14}$ V. V. Mitin, V. A. Kochelap, and M. A. Stroscio, Quantum Heterostructures (Microelectronics and Optoelectronics) (Cambridge University Press, New York, 1999).

${ }^{15}$ S. K. Jung, C. K. Hyon, J. H. Park, S. W. Hwang, D. Ahn, M. H. Son, B. D. Min, Y. Kim, and E. K. Kim, Phys. Lett. 75, 8 (1999).

${ }^{16}$ R. Wetzler, A. Wacker, E. Schöll, C. M. A. Kapteyn, R. Heitz, and D. Bimberg, Appl. Phys. Lett. 77, 1671 (2000).

${ }^{17}$ P. N. Brounkov, A. Polimeni, S. T. Stoddart, M. Henini, L. Eaves, P. C. Main, A. R. Kovsh, Yu. G. Mushikhin, and S. G. Konnikov, Appl. Phys. Lett. 73, 1092 (1998).

${ }^{18}$ A. Haggag and K. Hess, IEEE Trans. Electron Devices 47, 1624 (2000).

${ }^{19}$ M. Shur, Physics of Semiconductor Devices (Wiley, New York, 1981), p. 152.

${ }^{20}$ S. M. Sze, Physics of Semiconductor Devices (Wiley, New York, 1981), p. 89.

${ }^{21}$ M. Shur, in Ref. 19 , p. 154

${ }^{22}$ J. G. Ma, K. S. Yeo, and M. A. Do, IEEE Trans. Electron Devices 46, 2357 (1999).

${ }^{23}$ M. Ershov, H. C. Liu, L. Li, Buchanan, Z. R. Wasilewski, and K. Jonscher, IEEE Trans. Electron Devices 45, 2196 (1998).

${ }^{24}$ H. W. Li and T. H. Wang, Appl. Phys. A: Mater. Sci. Process. , 1007 (2001) 